\title{
CLINICAL PITFALLS OF LEISHMANIASIS AND WHIPPLE'S DISEASE HIDDEN BEHIND SYSTEMIC LUPUS ERYTHEMATOSUS: A CASE SERIES
}

\author{
Francesca losa, Davide Firinu*, Margherita Deidda, Giulia Costanzo \\ and STEFANO R. DEL GIACCO
}

Department of Medical Sciences and Public Health and Unit of Allergy and Clinical Immunology, University Hospital "Duilio Casula", University of Cagliari, Monserrato (Cagliari), Italy

(Received: 22 January 2019; accepted: 4 March 2019)

\begin{abstract}
Systemic lupus erythematosus (SLE) is a multisystemic autoimmune disease that can affect major organs possibly leading to life-threatening complications and appears with heterogeneous clinical picture. SLE could present with broad spectrum of clinical and laboratory features that can resemble those of other diseases, such as hemopoietic malignancies, infections, or immune-mediated disorders. Its complexity and protean features overlap with many other diseases, hindering the differential diagnosis. Rarely, true overlap with other diseases may occur. Herein, we report a case series of two patients affected by infectious diseases, namely visceral leishmaniasis and Whipple's disease (WD), intertwined with clinical or serological features of SLE. In both cases, several confounding factors have led to a delay in the diagnosis. Moreover, we first describe the persistent elevation of autoantibodies and a monoclonal gammopathy in a patient with WD. Awareness of unusual presentations of infections or other rare disorders, which may be encountered in clinical practice when taking care of SLE patients, is essential for timely diagnosis and treatment of potentially lethal diseases.
\end{abstract}

Keywords: systemic lupus erythematous, visceral leishmaniasis, Whipple's disease, monoclonal gammopathy of undetermined significance, fever, splenomegaly, aplasia

\section{Introduction}

Systemic lupus erythematosus (SLE) is a multisystemic autoimmune disease that can affect major organs possibly leading to life-threatening complications and presents with a heterogeneous clinical picture. Despite many studies have investigated its causes, the etiology remains poorly understood [1].

\footnotetext{
*Corresponding author; E-mail: davide.firinu@unica.it
} 
SLE more frequently affects women than men (ratio 9:1), primarily during reproductive periods. The most widely used classification criteria for SLE were developed by the American College of Rheumatology (ACR) [2, 3]. A patient has to fulfill at least 4 of these 11 criteria to be classified as having SLE.

SLE could present with broad spectrum of clinical and laboratory features that can resemble those of other diseases, such as hemopoietic malignancies, infections, or immune-mediated disorders. Moreover, the clinical picture can be complicated by manifestations due to drug side effects, infections, and other comorbid disorders. On the other hand, various diseases can mimic SLE flares, making the differential diagnosis difficult. The misdiagnosis of these conditions may cause delays in appropriate treatment and lead to the inadequate use of immunosuppressive drugs with potentially dangerous effects [4].

SLE is a disease known as one of the "great imitators": its complexity and protean features overlap with many other diseases, hindering the differential diagnosis. Rarely, true overlap with other diseases may occur [5, 6].

Herein, we report two patients affected by rare infectious disorders intertwined with SLE.

\section{Case report 1}

A 52-year-old woman was referred to our department presenting proteinuria $(1.53 \mathrm{~g} / 24 \mathrm{~h})$ since 3 months and fever, intense fatigue, and migrant arthritis at the referral time. Laboratory workup evidenced anemia, thrombocytopenia, elevated erythrocyte sedimentation rate (ESR) and C-reactive protein levels, positivity of antinuclear antibody (ANA) 1:160 with homogeneous pattern, and anti-ds-DNA antibody to $45.3 \mathrm{IU} / \mathrm{ml}$ (Farr assay). According to the ACR criteria, she was diagnosed with SLE and initially treated with intravenous methylprednisolone pulses and then prednisone $(25 \mathrm{mg})$ daily associated with hydroxychloroquine $(400 \mathrm{mg})$ daily. Since partial clinical and laboratory response was achieved after 2 months, the patient underwent intravenous cyclophosphamide (CYC) pulses (750 mg monthly). Ten days after the first pulse, she was admitted to our wards presenting fever $\left(39^{\circ} \mathrm{C}\right.$ peak), severe thrombocytopenia $(8,000 / \mu 1)$, severe neutropenia $(300 / \mu \mathrm{l})$, anemia [hemoglobin $(\mathrm{Hb}) 6.6 \mathrm{~g} / \mathrm{dl}$ ], and hepatosplenomegaly. An empirical therapy with different combinations of broad-spectrum antibiotics (levofloxacin, ceftriaxone, meropenem, gentamicin, teicoplanin, clarithromycin, and linezolid) was started together with antifungal therapy (fluconazole). Iatrogenic bone marrow aplasia was suspected; thus, immunosuppressive treatment was discontinued and the patient underwent blood and platelet transfusions and administrations of lenograstim (recombinant human G-CSF). No bone marrow biopsy was performed, as the consultant 
hematologist was confident of an iatrogenic cause of aplasia. Due to persistence of fever, intravenous amphotericin B substituted fluconazole after 15 days. After 20 days of aplasia, her condition was improved, with decrease of fever and gradual recovery of bone marrow function. The patient was discharged afebrile with greatly improved conditions. Immunosuppressive therapy for SLE was reintroduced with mycophenolate mofetil $2,000 \mathrm{mg}$ /day and oral prednisone.

Two months later, the patient started to present fever and fatigue. The persistence of hepatosplenomegaly was observed and serum protein electrophoresis revealed a suspected monoclonal gammopathy, not documented before. Urinary immunofixation revealed a monoclonal component of kappa free-light chains and serum immunofixation a monoclonal component IgG-K. Bone marrow aspirate and biopsy showed the presence of normal erythroid and myeloid series; mature lymphocytes $<10 \%$, plasma cells and blast proportion $<5 \%$, but unexpectedly there was a striking presence of small elements within the cytoplasm of histiocytes and macrophages consistent with leishmania infestation (Figure 1). High titers of serum anti-leishmania antibodies were demonstrated ( $\geq 30$ antibody units).

Immunosuppressive therapy was discontinued and high dose of liposomal amphotericin B, i.e., $3 \mathrm{mg} / \mathrm{kg}$ (total dose $=1,400 \mathrm{mg}$ ), was administered. In the following 3 weeks, fever and weakness gradually disappeared and inflammatory markers normalized. The monoclonal component gradually decreased until it disappeared after approximately 1 year as well as autoantibody positivity.

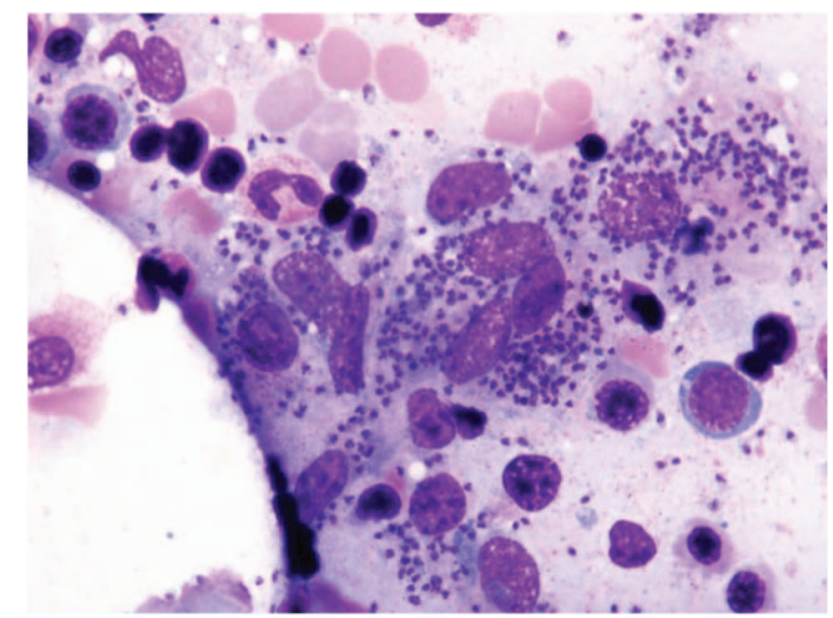

Figure 1. Leishmania phagocytosed by the macrophages in bone marrow blood smear 
Case report 2

A 30-year-old woman presented with 1-year history of $7 \mathrm{~kg}$ weight loss, fever, and night sweats. In 2006, she was diagnosed with epithelioid granulomatous necrotizing lymphadenopathy. In 2011, a gastroenteritis by Salmonella species, and hypochromic microcytic anemia resistant to iron therapy had been diagnosed and treated. Physical examination revealed lymph nodes of about $1 \mathrm{~cm}$ in the right cervical area. Laboratory values were as follows: $\mathrm{Hb} 8.2 \mathrm{~g} / \mathrm{dl}$; elevated ESR to $54 \mathrm{~mm} / \mathrm{h}$, ANA positive in a titer 1:640, anti-ds-DNA $85.1 \mathrm{UI} / \mathrm{ml}$, and RNP $33 \mathrm{UI} / \mathrm{ml}$. Serum immunofixation revealed a monoclonal IgG-lambda component. Computed tomography imaging showed hepatomegaly and multiple enlarged and confluent lymph nodes of the hepatic hilum, para-aortic, mesenteric, and iliac region. Bone marrow aspirate and biopsy, cancer markers, colonoscopy, screening for celiac disease, quantiferon, fecal cultures, and calprotectin levels were normal. Suspecting a lymphoproliferative disorder, a laparoscopic abdominal lymph node biopsy was performed. Histology showed aggregates of foamy periodic acid-Schiff positive and diastase-resistant histiocytes containing abundant cytoplasmatic granular material, compatible with Whipple's disease (WD). Analysis by polymerase chain reaction (PCR) of 16S rRNA of biopsy tissue confirmed the diagnosis [7]. Revision of the cervical lymph node biopsy performed in 2006 was also suggestive of WD. The esophagogastroduodenoscopy and bowel biopsies of the upper gastrointestinal tract were normal.

Therapy with intravenous ceftriaxone and then with oral sulfamethoxazole/ trimethoprim was established. One month after the antibiotic therapy, the general conditions, inflammatory markers, and complete blood counts started to improve. Furthermore, after 6 months, a thyroid biopsy showed the presence of a papillary carcinoma. Total thyroidectomy was performed. To date, the partial normalization of antibody values has been documented after 18 months of antibiotic therapy: $\mathrm{Hb}$ $12.2 \mathrm{~g} / \mathrm{dl}$, ESR $30 \mathrm{~mm} / \mathrm{h}$, ANA positive in a titer 1:640, anti-ds-DNA to $8.0 \mathrm{UI} / \mathrm{ml}$ (normal reference $<7 \mathrm{IU} / \mathrm{ml}$ ), and anti-RNP to $17 \mathrm{UI} / \mathrm{ml}$. Monoclonal component is still detectable.

\section{Discussion}

Several studies have investigated the role of infectious agents in the pathogenesis of SLE, hypothesizing that the disease may be the consequence of an uncontrolled immune response against pathogens in susceptible individuals $[8,9]$. Molecular mimicry, superantigens, epitope spreading, bystander activation, and activation of lymphocytes by lymphotropic viruses are commonly suggested 
as main mechanisms involved in the triggering of the autoimmune response [10]. The association of viral infections for the production of autoantibodies and lupuslike disorders has been widely reported in literature [11]. Most of the evidences are available for Epstein-Barr virus [12], but some authors suggested that parvovirus B19, retrovirus such as Human T-lymphotropic virus type-1 and cytomegalovirus might be involved in the pathogenesis [13-16].

SLE is an autoimmune disease characterized by B-cell activation, which plays a crucial pathological role through the presentation of self-antigens, T-cell activation, and the production of autoantibodies and cytokines [17]. Abnormal B-cell function is characterized by the production of a wide range of autoantibodies, the serologic hallmark of SLE. Another characteristic of pathological B-cell function is cytokine secretion: B cells produce interleukin-4, 6, 10, interferon gamma, transforming growth factor beta, and lymphotoxin-alpha, but in SLE, overproduction of cytokines can lead to amplification of autoimmune response. Monoclonal gammopathy of undetermined significance (MGUS) is defined as the presence of a monoclonal immunoglobulin (M-protein or M-component) in the serum or urine of an individual with no evidence of multiple myeloma, amyloid light-chain amyloidosis, Waldenström macroglobulinemia, or other related disorders. A terminally differentiated clone of B cells secretes the monoclonal component. MGUS is frequently associated with various connective diseases, such as rheumatoid arthritis, SLE, scleroderma, and various infective agents, such as hepatitis $C$ virus, hepatitis B virus, human immunodeficiency virus, and Helicobacter pylori. However, there is no clear evidence for a pathogenetic role of the M-protein [18].

Visceral leishmaniasis (VL) is a parasitic disease transmitted to humans by sandflies in endemic areas and considered endemic also in Mediterranean basin. In Italy, the highest incidence is in the southern regions, such as Sardinia. VL typically presents with fever, severe anorexia, hepatosplenomegaly, while laboratory tests show cytopenias, hypergammaglobulinemia, and elevation of acute phase markers [19].

Autoimmune manifestations are common in VL secondary to strong T-cell and B-cell responses. High levels of circulating immune complexes and selfantigen are common in VL: this might be due to a release of high amounts of antigens, as a result of tissue destruction, that may stimulate the autoreactivity and autoantibody production. Polyclonal B-cell hyperactivation results in the severe hypergammaglobulinemia that can be observed in VL.

VL often remains asymptomatic but may have a life-threatening course with fever, anorexia, hepatosplenomegaly, and kidney involvement [20].

VL has been associated with multiple immunological abnormalities and owing to its protean clinical manifestations may be misinterpreted as various autoimmune 
diseases [21]. Moreover, response to immunosuppressive treatments may masquerade an undergoing VL. Serum antibodies such as ANA and anti-ds-DNA, low serum C3 and C4 complements levels, and positive direct Coombs' reaction are the common SLE-related immunological findings that have been reported in the course of VL [22]. Thus, these elements might lead to misdiagnosis of VL for SLE.

SLE and many other autoimmune diseases may have clinical and laboratory features overlapping with VL that could hinder the right diagnosis: both can present with fever, splenomegaly, pericardial effusion, and pancytopenia. Moreover, many autoantibodies formed in VL disease are commonly specific in different autoimmune systemic and organ-specific diseases: anti-ds-DNA in SLE or anti-smooth muscle antibody in autoimmune hepatitis [13, 23].

A correct and early diagnosis of VL in our patient was quite difficult; initial response to immunosuppressive therapy further supported the SLE diagnosis. Hematological manifestations appeared later in the clinical course and were at first considered as a CYC side effect. Cytopenias are common in SLE, due to either bone marrow failure, excessive peripheral cell destruction, or adverse reactions to medication. Treatment involves steroids, steroid-sparing agents, and splenectomy [24]. However, in our patient, discontinuation of the drugs did not completely resolve the clinical picture, and the persistence of proteinuria and the appearance of monoclonal gammopathy led us to reevaluate the case revealing VL diagnosis. This is a finding reported in recent years in VL patients [25].

The emergence of monoclonal components in the course of autoimmune diseases or in young people deserves special attention, in order to exclude a wide range of diseases or to start an adequate follow-up program [18]. The second case reported shares the previous case of the occurrence of a long-lasting monoclonal gammopathy, in a patient affected by WD. This occurrence has not been reported in the literature, as well as the elevation of ANA, anti-ds-DNA, and RNP antibody, which are classically observed in SLE. We did not diagnose the patient as having SLE, mainly due to the lack of consistent clinical manifestations of the disease. However, this will not preclude that the patient (a young woman) may eventually develop further features of SLE in the future. It is relevant to note that antibodies showed a slow trend toward normalization, even if a larger timeframe of observation is required.

WD is a chronic bacterial infection often characterized by malabsorption syndrome with diarrhea and weight loss, fever, and lymphadenopathy [26]. Cases of WD negative at bowel biopsies are documented [27]. The analysis of 16S rRNA or novel PCR methods on biopsies and samples is essential to confirm the diagnosis in WD or in other rare infectious diseases [7, 28].

A single case of WD and SLE has been described to date [29]. Interestingly, although clear pathological elements of WD are recognizable, non-specific 
histopathological and clinical presentations are known [30]. Further observations and studies may be useful to gain insight from the interaction between the immune system and Tropheryma whipplei, which is pivotal as demonstrated by the immune reconstitution syndrome that often arises after initializing WD treatment. To our knowledge, this is the first report of the coexistence between WD and persistent elevation of autoantibodies and the detection of a monoclonal gammopathy.

In conclusion, the awareness of unusual presentations of infections, which may be encountered in the daily clinical practice when taking care of SLE patients, may be essential for timely diagnosis and proper treatment of such manifold and potentially lethal diseases.

\section{Acknowledgements}

The data sets used and/or analyzed during this study are available from the corresponding author on reasonable request. LF, GC, and DF were responsible for the data analysis and interpretation and contributed to writing of the manuscript. DF and SRdG were responsible for the data selection and clinical interpretation of the data. DF designed the study and interpreted the data. All authors read and approved the final version of the manuscript. The patients gave written consent; however, the authors made efforts to remove identifying information to protect the anonymity of the patients.

\section{Conflict of Interest}

The authors declare no competing interests.

\section{References}

1. Rahman, A., Isenberg, D. A.: Systemic lupus erythematosus. N Engl J Med 358, 929-939 (2008).

2. Tan, E. M., Cohen, A. S., Fries, J. F., Masi, A. T., McShane, D. J., Rothfield, N. F., Schaller, J. G., Talal, N., Winchester, R. J.: The 1982 revised criteria for the classification of systemic lupus erythematosus. Arthritis Rheum 25, 1271-1277 (1982).

3. Hochberg, M. C.: Updating the American College of Rheumatology revised criteria for the classification of systemic lupus erythematosus. Arthritis Rheum 40, 1725 (1997).

4. Bertsias, G., Ioannidis, J. P., Boletis, J., Bombardieri, S., Cervera, R., Dostal, C., Font, J., Gilboe, I. M., Houssiau, F., Huizinga, T.: EULAR recommendations for the management of systemic lupus erythematosus. Report of a Task Force of the EULAR Standing Committee 
for International Clinical Studies Including Therapeutics. Ann Rheum Dis 67, 195-205 (2008).

5. Jansen, A. F. M., Raijmakers, R. P. H., Keijmel, S. P., van der Molen, R. G., Vervoort, G. M., van der Meer, J. W. M., van Deuren, M., Bleeker-Rovers, C. P.: Autoimmunity and B-cell dyscrasia in acute and chronic Q fever: A review of the literature. Eur J Intern Med 54, 6-12 (2018).

6. Murgia, G., Firinu, D., Meleddu, R., Lorrai, M. M., Manconi, P. E., Del Giacco, S. R.: Systemic lupus erythematosus occurring in a patient with Niemann-Pick type B disease. Lupus 24, 1332-1334 (2015).

7. Gras, E., Matias-Guiu, X., Garcia, A., Argüelles, R., Espinosa, I., Sancho, F. J., Sola, R., Martinez-Araque, M. J., Conde, J., Teruel, A., Prat, J.: PCR analysis in the pathological diagnosis of Whipple's disease: Emphasis on extraintestinal involvement or atypical morphological features. J Pathol 188, 318-321 (1999).

8. Steri, M., Orrù, V., Idda, M. L., Pitzalis, M., Pala, M., Zara, I., Sidore, C., Faà, V., Floris, M., Deiana, M.: Overexpression of the cytokine BAFF and autoimmunity risk. N Engl J Med 376, 1615-1626 (2017).

9. Cooper, G. S., Dooley, M. A., Treadwell, E. L., St Clair, E. W., Gilkeson, G. S.: Risk factors for development of systemic lupus erythematosus: Allergies, infections, and family history. J Clin Epidemiol 55, 982-989 (2002).

10. Rigante, D., Mazzoni, M. B., Esposito, S.: The cryptic interplay between systemic lupus erythematosus and infections. Autoimmun Rev 13, 96-102 (2014).

11. Samarkos, M., Vaiopoulos, G.: The role of infections in the pathogenesis of autoimmune diseases. Curr Drug Targets Inflamm Allergy 4, 99-103 (2005).

12. Draborg, A. H., Duus, K., Houen, G.: Epstein-Barr virus in systemic autoimmune diseases. Clin Dev Immunol 2013, 535738 (2013).

13. Pavlovic, M., Kats, A., Cavallo, M., Shoenfeld, Y.: Clinical and molecular evidence for association of SLE with parvovirus B19. Lupus 19, 783-792 (2010).

14. Quaresma, J. A., Yoshikawa, G. T., Koyama, R. V., Dias, G. A., Fujihara, S., Fuzii, H. T.: HTLV-1, immune response and autoimmunity. Viruses 8, 5 (2015)

15. Hayashi, T., Lee, S., Ogasawara, H., Sekigawa, I., Iida, N., Tomino, Y., Hashimoto, H., Hirose, S.: Exacerbation of systemic lupus erythematosus related to cytomegalovirus infection. Lupus 7, 561-564 (1998).

16. Nawata, M., Seta, N., Yamada, M., Sekigawa, I., Lida, N., Hashimoto, H.: Possible triggering effect of cytomegalovirus infection on systemic lupus erythematosus. Scand J Rheumatol 30, 360-362 (2001).

17. Putterman, C., Caricchio, R., Davidson, A., Perlman, H.: Systemic lupus erythematosus. Clin Dev Immunol 2012, 437282 (2012).

18. Kyle, R. A., Therneau, T. M., Rajkumar, S. V., Larson, D. R., Plevak, M. F., Offord, J. R., Dispenzieri, A., Katzmann, J. A., Melton, L. J.: Prevalence of monoclonal gammopathy of undetermined significance. N Engl J Med 354, 1362-1369 (2006).

19. van Griensven, J., Diro, E.: Visceral leishmaniasis. Infect Dis Clin North Am 26, 309-322 (2012).

20. Verma, N., Lal, C. S., Rabidas, V., Pandey, K., Singh, D., Kumar, S., Verma, R. B., Das, P.: Microalbuminuria and glomerular filtration rate in paediatric visceral leishmaniasis. Biomed Res Int 2013, 498918 (2013). 
21. Bogdan, C.: Leishmaniasis in rheumatology, haematology and oncology: Epidemiological, immunological and clinical aspects and caveats. Ann Rheum Dis 71, i60-66 (2012).

22. Voulgarelis, M., Voulgari, P. V., Serelis, J., Drosos, A. A., Skopouli, F. N.: Visceral leishmaniasis resembling systemic lupus erythematosus. Clin Rheumatol 22, 452-455 (2003).

23. Sakkas, L. I., Boulbou, M., Kyriakou, D., Makri, I., Sinani, C., Germenis, A., Stathakis, N.: Immunological features of visceral leishmaniasis may mimic systemic lupus erythematosus. Clin Biochem 41, 65-68 (2008).

24. Sinha, P. K., van Griensven, J., Pandey, K., Kumar, N., Verma, N., Mahajan, R., Kumar, P., Kumar, R., Das, P., Mitra, G.: Liposomal amphotericin B for visceral leishmaniasis in human immunodeficiency virus-coinfected patients: 2-year treatment outcomes in Bihar, India. Clin Infect Dis 53, e91-e98 (2011).

25. Rombola, F., Spinoso, A., Bertuccio, S. N.: Monoclonal gammopathy after visceral leishmaniasis: Just a coincidence? Infez Med 16, 173-174 (2008).

26. Schneider, T., Moos, V., Loddenkemper, C., Marth, T., Fenollar, F., Raoult, D.: Whipple's disease: New aspects of pathogenesis and treatment. Lancet Infect Dis 8, 179-190 (2008).

27. Bruhlmann, P., Michel, B.A., Altwegg, M.: Diagnosis and therapy monitoring of Whipple's arthritis by polymerase chain reaction. Rheumatology 39, 1427-1428 (2000).

28. Appak, Ö., Türkel, S., Esen, N., Özkütük, A.A.: Comparison of polymerase chain reactionrestriction enzyme analysis method and DNA sequence analysis results in the identification of non-tuberculous mycobacteria. Acta Microbiol Immunol Hung 65, 515-527 (2018).

29. Ehrenfeld, M., Urowitz, M. B., Platts, M. E.: Selective C4 deficiency, systemic lupus erythematosus, and Whipple's disease. Ann Rheum Dis 43, 91-94 (1984).

30. Arnold, C. A., Moreira, R. K., Lam-Himlin, D., De Petris, G., Montgomery, E.: Whipple disease a century after the initial description: Increased recognition of unusual presentations, autoimmune comorbidities, and therapy effects. Am J Surg Pathol 36, 1066-1073 (2012). 